\title{
Microeconomic Origins of Macroeconomic Tail Risks*
}

\author{
Daron Acemoglu ${ }^{\dagger}$
}

\author{
Asuman Ozdaglar $\ddagger$
}

This version: December 2014

First version: June 2013
Alireza Tahbaz-Salehi ${ }^{\S}$

\begin{abstract}
We document that even though the normal distribution is a good approximation to the nature of aggregate fluctuations, it severely underpredicts the frequency of large economic downturns. We then provide a model that can explain these facts simultaneously. Our model shows that the propagation of microeconomic shocks through input-output linkages can fundamentally reshape the distribution of aggregate output, increasing the likelihood of large downturns (macroeconomic tail risks) from infinitesimal to substantial. For example, an economy subject to thin-tailed micro shocks but with "unbalanced" input-output linkages (where some sectors or firms play a much more important role than others as inputs suppliers to the rest of the economy) may exhibit deep recessions as frequently as economies that are subject to heavy-tailed shocks. This is despite the fact that a central limit theorem-type result would imply that aggregate output is normally distributed. We characterize what types of input-output linkages and distributions of microeconomic shocks lead to sizable macroeconomic tail risks, and also show how the same economic forces cause the output of many sectors to simultaneously fall by large amounts.
\end{abstract}

Keywords: Business cycles, macroeconomic tail risks, input-output linkages. JEL Classification: C67, E32.

${ }^{*}$ This paper is partially based on the previously unpublished results in the working paper of Acemoglu, Ozdaglar, and Tahbaz-Salehi (2010). An earlier draft of this paper was circulated under the title "the network origins of large economic downturns.” We are grateful to Vasco Carvalho, Xavier Gabaix, Mihalis Markakis, Emi Nakamura, Jón Steinsson and José Zubizarreta for fruitful conversations. Acemoglu and Ozdaglar gratefully acknowledge financial support from the Army Research Office, Grant MURI W911NF-12-1-0509.

${ }^{\dagger}$ Department of Economics, Massachusetts Institute of Technology.

${ }^{\ddagger}$ Laboratory for Information and Decision Systems, Massachusetts Institute of Technology.

${ }^{\S}$ Columbia Business School, Columbia University. 


\section{Introduction}

Most empirical studies in macroeconomics approximate the deviations of aggregate economic variables from their trends with a normal distribution. Besides its relative success in capturing salient features of the behavior of aggregate variables in the US and other OECD countries, this approach has a natural justification: since most macro variables (such as GDP) are obtained from combining more disaggregated ones, it is reasonable to expect that a central limit theorem-type result should imply that such macro variables are normally distributed. As an implicit corollary to this observation, most of the literature treats the standard deviations of aggregate variables as sufficient statistics for measuring aggregate economic fluctuations.

That the normal distribution is, for the most part, a good approximation to the nature of aggregate fluctuations can be seen from panels (a) and (b) of Figure 1. These panels depict the quantilequantile (Q-Q) plots of the US postwar quarterly output growth and the HP-detrended GDP against the standard normal distribution, while excluding tail risks or "large deviations" from the sample defined as quarters in which GDP growth or detrended output were above the top 5\% or below the bottom $5 \%$ of their respective empirical distributions. The close correspondence between the normal distribution, shown as the dashed red line, and the two truncated data series shows that, once large deviations are excluded, the normal distribution is indeed a good candidate for approximating GDP fluctuations. ${ }^{1}$

This picture, however, changes dramatically once large deviations are also taken into account. Panels (c) and (d) of Figure 1 show the same quantile-quantile plots for the entire US postwar sample. It is easy to notice that both graphs exhibit sizable and systematic deviations from the normal line at both ends. ${ }^{2}$ This observation highlights that even though the normal distribution does a fairly good job in approximating the nature of fluctuations during most of the sample, it severely underestimates the most consequential fact about business cycle fluctuations, namely, the frequency of large economic contractions.

Motivated by these patterns, this paper makes two related points. First, it argues that even though GDP is obtained from aggregating microeconomic variables, there is no guarantee that it should be normally distributed - even in the absence of aggregate shocks. Rather, input-output linkages within the economy can reshape the distribution of aggregate variables. Thus, in general, the standard deviation of aggregate variables may not be a sufficiently informative statistic for the

\footnotetext{
${ }^{1}$ To test for this claim formally, we first excluded the top and bottom $5 \%$ of observations from US GDP growth and detrended log GDP between 1947:Q1 and 2013:Q3, and then performed the Anderson-Darling and Jarque-Bera tests of normality. The resulting test statistics for GDP growth are 0.65 and 0.99 , respectively, and the test statistics for detrended (with HP filter 1600) log GDP are, respectively, 0.31 and 0.85 . In none of these cases we can reject normality at the $10 \%$ significance level.

${ }^{2}$ Repeating the tests in footnote 1 for the full sample of log GDP growth and detrended quarterly GDP leads to a strong rejection of normality in both cases: the Anderson-Darling and Jarque-Bera tests yield, respectively, test statistics of 1.91 and 21.6 for GDP growth, hence rejecting normality at the $0.5 \%$ level. Similarly for the detrended quarterly GDP, the two tests lead to test statistics of 1.98 and 33.1 , again rejecting normality at the $0.5 \%$ level.
} 


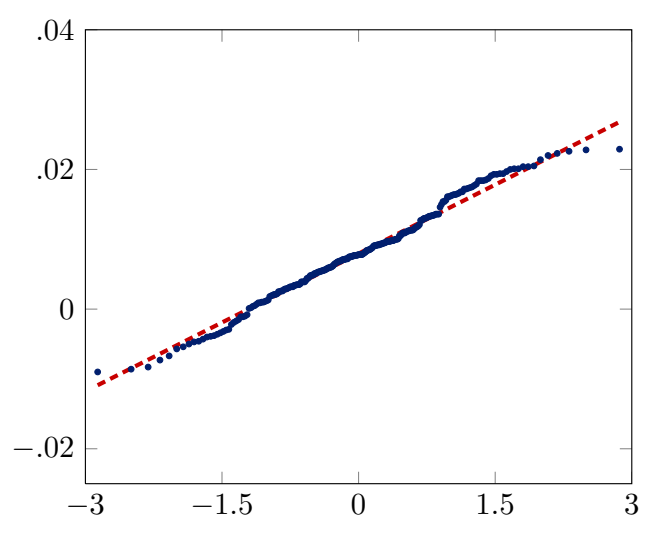

(a) truncated GDP growth rate

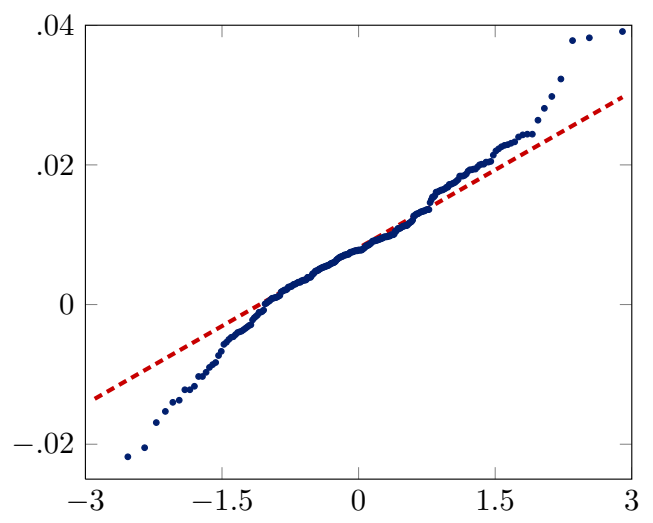

(c) GDP growth rate

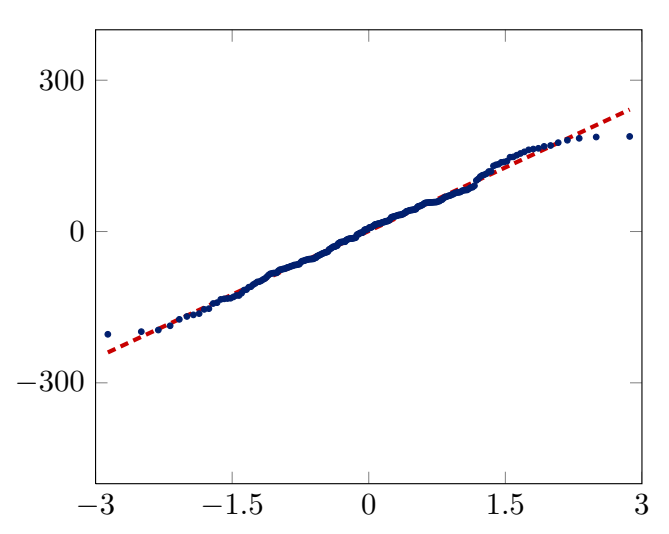

(b) HP-detrended and truncated output

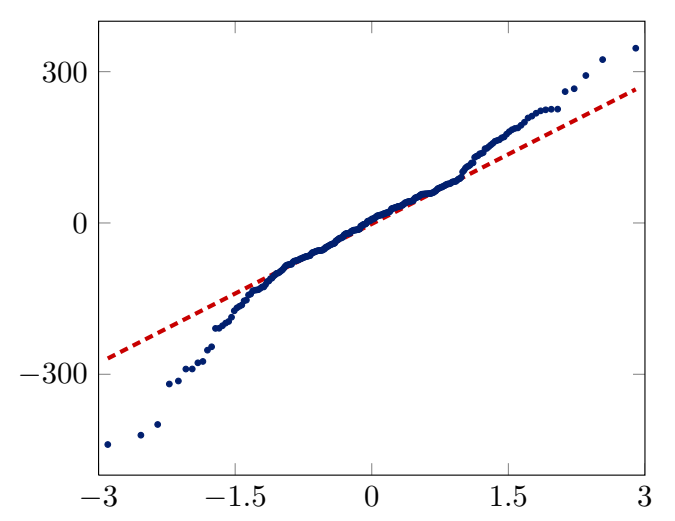

(d) HP-detrended output

Figure 1. The quantile-quantile (Q-Q) plots of the postwar U.S. GDP fluctuations (1947:QI to 2013:QIII) versus the standard normal distribution, shown by the dashed red line. The horizontal and vertical axes correspond, respectively, to the quantiles of the standard normal distribution and the sample data. Panels (a) and (b) depict the Q-Q plots for the GDP growth rate and HP-detrended output. The linearity of the points suggests that both truncated datasets are approximately normally distributed. Panels (c) and (d) depict the Q-Q plots of the two datasets after removing the top and bottom 5\% of data points. The deviation from the dashed red line suggests that both datasets exhibit heavier tails compared to the normal distribution.

nature of aggregate fluctuations. Second, we argue that the failure of normality manifests itself in the form of significantly higher "tail risks". In particular, we show that the propagation of microeconomic shocks through input-output linkages can lead to more frequent and larger economic downturns than what is predicted by the normal distribution. We also demonstrate that the emergence of such macroeconomic tail risks can coexist with approximately normally distributed fluctuations away from the tails, consistent with the pattern of US GDP fluctuations documented in Figure 1.

Though there are many different modeling approaches that can generate significant tail risks (e.g., models with financial crises or multiple equilibria), in this paper, we focus on the role of input-output linkages across disaggregated subsectors along the lines of Long and Plosser (1983) and Acemoglu et al. (2012). This choice is motivated by two considerations. First, a model consisting of many sectors subject to idiosyncratic shocks is a natural starting point for generating aggregate fluctuations that are reasonably well-approximated by a normal distribution. Second, as 
shown in Acemoglu et al. (2012), certain types of input-output linkages can generate significant aggregate volatility (and comovement across sectors) from idiosyncratic microeconomic shocks. They are thus also a potential candidate for generating large deviations or tail risks.

In order to develop the link between the nature of macroeconomic tail risks, microeconomic shocks, and the input-output structure of the economy, we first study the class of balanced economies as a benchmark. These are economies in which all sectors play roughly symmetric roles as input suppliers to others. For this class of economies, we show that, regardless of the distribution of microeconomic shocks, not only GDP fluctuations are normally distributed, but also large economic downturn are exponentially unlikely. In other words, absent any other amplification mechanisms or aggregate shocks - and in contrast to the patterns observed in Figure 1 - the likelihood of large contractions in such economies is infinitesimal. Furthermore, we show that the standard deviation of log GDP can serve as a sufficient statistic for the likelihood of large downturns.

Our subsequent analyses, however, establish that the above mentioned results for balanced economies do not hold for general economies. More specifically, the implications of our theoretical results can be summarized as follows:

First, the propagation of microeconomic shocks through input-output linkages in an unbalanced economy - where some sectors play a much more important role than others as inputs suppliers to the rest of the economy - can lead to the emergence of significant macroeconomic tail risks. Furthermore, we show that the frequency of large GDP contractions is highly sensitive to the nature of microeconomic shocks. In particular, micro shocks with slightly thicker tails can lead to a significant increase in the likelihood of large economic downturns.

Second, depending on the distribution of microeconomic shocks (and in contrast to the case of balanced economies), the economy may exhibit significant macroeconomic tail risks even though aggregate fluctuations away from the tails can be well-approximated by a normal distribution, an outcome consistent with the pattern of US postwar GDP fluctuations documented in Figure 1. This result is a consequence of the fact that the propagation of shocks over input-output linkages can concentrate the risks at the tails of the distribution.

Third, our results imply that the standard deviation of aggregate variables (such as log GDP) may no longer serve as a sufficient statistic for the likelihood of large economic downturns: two economies subject to micro shocks drawn from different distributions may experience large recessions with significantly different frequencies, even when their GDP fluctuations have identical standard deviations. The juxtaposition of our last two results underscores the importance of studying the determinants of large recessions, since such macroeconomic tail risks may vary significantly even across economies that exhibit otherwise identical behavior for moderate deviations.

Fourth, to highlight the key role of input-output linkages in creating macroeconomic tail risks, we show that an economy with unbalanced inputs-output linkages subject to thin-tailed (e.g., exponentially distributed) microeconomic shocks exhibits deep recessions as frequently as a balanced 
economy subject to heavy-tailed (e.g., Pareto distributed) shocks. In this sense, our results provide a novel solution to what Bernanke, Gertler, and Gilchrist (1996) refer to as the "small shocks, large cycles puzzle" by arguing that the interaction between the underlying input-output structure of the economy and the shape of the distribution of microeconomic shocks is of first-order importance in determining the nature of aggregate fluctuations.

Finally, we demonstrate that the forces identified above not only lead to the emergence of macroeconomic tail risks, but can also generate "tail comovements," defined as simultaneous large falls in the output of many sectors. This type of comovement is akin to the effects of aggregate shocks.

We conclude the paper with a simple quantitative exercise highlighting the role of input-output linkages in the emergence of macroeconomic tail risks. Using the input-output matrix of the US economy, we show that when microeconomic shocks are drawn from a distribution with slightly thicker tails than the normal distribution (such as the exponential distribution), the pattern of aggregate fluctuations would be similar to Figure 1: generally close to normal distribution but with significantly larger likelihood of tail risks. In contrast, if microeconomic shocks are normally distributed, input-output linkages have no effect on the likelihood and frequency of large economic downturns.

Related Literature Our paper belongs to the small literature that focuses on large macroeconomic downturns. A number of papers, including Cole and Ohanian $(1999,2002)$ and Kehoe and Prescott (2002), have used the neoclassical growth framework to study Great Depression-type events in the United States and other countries. More recently, there has been a growing emphasis on deep Keynesian recessions due to liquidity traps and the zero lower bound on nominal interest rates (such as Christiano et al. (2011), Eggertsson and Krugman (2012), Eggertsson and Mehrotra (2014)). Relatedly, Christiano, Eichenbaum, and Trabandt (2015) argue that financial frictions can account for the key features of the recent economic crisis. Though our paper shares with this literature the emphasis on large economic downturns, both the focus and the underlying economic mechanisms are very different.

Our paper is also related to the literature on "rare disasters", such as Rietz (1988), Barro (2006), Gabaix (2012), Nakamura, Steinsson, Barro, and Ursúa (2013) and Farhi and Gabaix (2014), who argue that the possibility of rare but extreme disasters is an important determinant of risk premia in asset markets. Relaedly, Gourio (2012) studies a real business cycle model with a small risk of economic disaster. This literature, however, treats the frequency and the severity of such rare disasters as largely exogenous. In contrast, we provide a possible explanation for the endogenous emergence of such macroeconomic tail risks due to the propagation and amplification of microeconomic shocks. Furthermore, we characterize how the distributional properties of micro shocks coupled with the input-output linkages of the economy shape the likelihood and depth of large downturns.

Our paper is also closely related to the literature that studies the microeconomic origins of busi- 
ness cycle fluctuations. For example, Gabaix (2011) argues that if the firm size distribution is sufficiently heavy-tailed (in the sense that the largest firms contribute disproportionally to aggregate output), firm-level idiosyncratic shocks may translate into fluctuations at the aggregate level. Relatedly, Acemoglu et al. (2012) show that in the presence of input-output linkages, microeconomic shocks may have a non-trivial impact on the standard deviation of GDP. ${ }^{3}$ Clearly, our paper has much in common with Acemoglu et al. (2012), as we are using the same structure of microeconomic interactions impacting the macro economy through input-output linkages. Nevertheless, there are major differences between the two papers and also between the current work and the previous literature in general. First, rather than the standard deviation of GDP, the focus of the current paper is on the likelihood of large economic downturns which, to the best of our knowledge, is entirely new. Second, this shift in focus leads to a novel set of economic insights. In particular, our results establish that the standard deviation of GDP may not be an especially useful measure for the frequency and depth of large recessions, and also that it is the interplay between the shape of microeconomic shock distributions and the input-output linkages within the economy that is of first-order importance in determining the likelihood of such downturns, a result with no counterpart in the previous literature.

On the empirical side, Carvalho and Gabaix (2013) explore whether changes in the microeconomic composition of the economy during the post-war period can account for the Great Moderation, while Foerster, Sarte, and Watson (2011) and Atalay (2014) study the relative importance of aggregate and micro-level shocks in output volatility. Complementing these studies and using a database covering the universe of French firms, di Giovanni, Levchenko, and Méjean (2014) document that firm-level shocks contribute significantly to aggregate volatility and much of this contribution is driven by the presence of input-output linkages within the economy. Once again, however, this literature focuses predominantly on the standard deviation of GDP as the measure of aggregate fluctuations and is thus silent on the underlying reasons for the emergence of the patterns similar to the ones observed in Figure 1. We, on the other hand, argue that input-output linkages within the economy can explain the emergence of large output contractions despite the fact that aggregate output can be approximated by a normal distribution throughout most of the sample.

Finally, our paper is related to the growing literature that focuses on the role of power laws and large deviations in various contexts. For example, Gabaix et al. $(2003,2006)$ provide a theory of excess stock market volatility in which market movements are due to trades by very large institutional investors. Relatedly, Ibragimov et al. (2009) argue that when risk distributions have heavy tails, insurance providers may choose not to offer insurance for catastrophic risks. Unlike our paper, however, this literature treats such extreme events as largely exogenous and do not link them to macroeconomic tail risks.

\footnotetext{
${ }^{3}$ Earlier studies in this literature include Jovanovic (1987), Durlauf (1993), Horvath (1998, 2000), Dupor (1999) and Carvalho (2010).
} 
Outline of the Paper The rest of the paper is organized as follows. We present the model in Section 2. Our main results are presented in Sections 3 and 4. Section 5 contains a simple quantitative exercise, demonstrating the role of input-output linkages in the emergence of macroeconomic tail risks. Section 6 concludes. All proofs are provided in the Appendix.

\section{Theoretical Framework}

Our model is a static variant of the multi-sector model of Long and Plosser (1983), also analyzed by Acemoglu et al. (2012).

Consider a static economy consisting of a large number, $n$, of competitive sectors denoted by $\{1,2, \ldots, n\}$, each of which producing a distinct product. Each product can be either consumed by a mass of consumers or used as input for production of other goods. Firms in each sector employ Cobb-Douglas production technologies with constant returns to scale that transform labor and intermediate goods into final products. Sectoral production is subject to microeconomic productivity shocks, which we assume to be idiosyncratic and Harrod-neutral. ${ }^{4}$ More specifically, the output of sector $i$, which we denote by $y_{i}$, is equal to

$$
y_{i}=\left(A_{i} \ell_{i}\right)^{\beta} \prod_{j=1}^{n} x_{i j}^{w_{i j}},
$$

where $A_{i}$ is the aforementioned microeconomic productivity shock, $\ell_{i}$ is the amount of labor hired by the firms in sector $i, x_{i j}$ is the amount of good $j$ used for production of good $i$, and $\beta \in(0,1)$ is the share of labor in production. The exponent $w_{i j} \geq 0$ in (1) captures the share of good $j$ in the production technology of good $i$. In particular, a higher $w_{i j}$ means that good $j$ is more important in producing $i$, whereas $w_{i j}=0$ implies that good $j$ is not a required input for $i$ 's production technology. The assumption that firms employ constant returns to scale technologies implies that $\sum_{j=1}^{n} w_{i j}=1-\beta$ for all $i .^{5}$

We assume that the $A_{i}$ 's are independent and identically distributed across sectors. The independence assumption guarantees that the economy is not subject to aggregate shocks. We denote the common cumulative distribution function of $\epsilon_{i}=\log \left(A_{i}\right)$ by $F$, which we assume to be symmetric around the origin, to have full support over $\mathbb{R}$, and unless otherwise stated, to have finite moments. With some abuse of terminology, we refer to $\epsilon_{i}$ as the shock to sector $i$.

In addition to the firms, the economy is populated with a unit mass of identical consumers. Each consumer is endowed with one unit of labor which can be hired by the firms for the purpose of production. We assume that the representative consumer has logarithmic preferences over the $n$

\footnotetext{
${ }^{4}$ The focus on productivity shocks is for simplicity. In more elaborate versions of this setup, demand, credit or uncertainty shocks would play similar roles.

${ }^{5}$ This latter assumption is adopted for simplicity, and our results can be generalized to the case in which $\sum_{j=1}^{n} w_{i j}$ varies across $i$.
} 
goods produced in the economy. In particular,

$$
u\left(c_{1}, \ldots, c_{n}\right)=\sum_{i=1}^{n} \log \left(c_{i}\right),
$$

where $c_{i}$ is the amount of good $i$ consumed.

The competitive equilibrium of the economy described above is defined in the usual way: it consists of a collection of prices and quantities such that (i) the representative consumer maximizes her utility; (ii) the representative firm in each sector maximizes its profits while taking the prices and the wage as given; and (iii) all markets clear.

For the above economy, we summarize the intersectoral input-output linkages with matrix $W=$ $\left[w_{i j}\right]$, which we refer to as the input-output matrix of the economy. The corresponding Leontief inverse matrix of the economy is thus given by $L=(I-W)^{-1}$. We denote the effective share of industry $i$ 's output in the input supply of the entire economy by

$$
v_{i}=\sum_{j=1}^{n} L_{j i}
$$

which one can show is equal to the equilibrium share of sales of sector $i$ given by $v_{i}=p_{i} y_{i} / \sum_{j=1}^{n} p_{j} y_{j}$, where $p_{j}$ denotes the equilibrium price of good $j .{ }^{6}$ We thus refer to $v_{i}$ as the sales share of sector $i$. To see that $v_{i}$ does indeed correspond to the effective input share of industry $i$ 's output for the entire economy, note that it can be rewritten as ${ }^{7}$

$$
v_{i}=1+\sum_{j=1}^{n} w_{j i}+\sum_{j=1}^{n} \sum_{k=1}^{n} w_{j k} w_{k i}+\cdots
$$

which not only captures the direct impact of industry $i$ as an input supplier on its immediate downstream customers, but also its indirect impact on the customers of its customers and so on.

Throughout the paper, we focus on an infinite sequence of economies indexed by the number of sectors and study the distributional properties of aggregate output as $n \rightarrow \infty$, while taking other features of the economy (such as shock distribution $F$ and the share of labor in production $\beta$ ) fixed. The elements of this sequence can be interpreted as corresponding to different levels of disaggregation of the same economy.

Finally, throughout the paper, we refer to the logarithm of real value added in the economy as aggregate output and denote it by $y$.

\section{Microeconomic Origins of Macroeconomic Tail Risks}

In this section, we study how input-output linkages within the economy can explain the patterns observed in Figure 1. In particular, we argue that the interaction between input-output linkages and

\footnotetext{
${ }^{6}$ This is a consequence of theorem of Hulten (1978). A derivation of this result can be found in Acemoglu et al. (2012).

${ }^{7}$ This is an immediate consequence of the fact that $(I-W)^{-1}=\sum_{r=0}^{\infty} W^{r}$.
} 
the shape of the microeconomic shocks' distributions may fundamentally reshape the distribution of aggregate variables, leading to frequent and large output contractions despite the fact that GDP fluctuations, for the most part, can be approximated by a normal distribution.

We start our analysis by defining different notions that measure the severity and frequency of macroeconomic fluctuations. The most natural (and widely used) notion is the standard deviation of aggregate output, $\sigma_{y}=\operatorname{stdev}(y)$, which we simply refer to as aggregate volatility.

Definition 1. The aggregate volatility of the economy is trivial if $\sigma_{y} \sim 1 / \sqrt{n} .8$

The motivation for calling aggregate volatility "trivial" in this case is twofold. First, note that the statement $\sigma_{y} \sim 1 / \sqrt{n}$ essentially means that (idiosyncratic) microeconomic shocks wash out as predicted by the law of large numbers. As famously pointed out by Lucas (1977), in such a case, aggregate volatility originating from microeconomic shocks to highly disaggregated sectors would be negligible. Second, the standard deviation of (log) GDP in any such economy is observationally equivalent to an economy with no input-output linkages. ${ }^{9}$

Even though aggregate volatility is the most natural and widely used measure of the extent of fluctuations, because it is essentially the second-order moment of the distribution of GDP, it is not necessarily informative about the nature of large deviations. In fact, Figure 1 and the related discussion in the Introduction highlight the need for a separate measure that can capture the extent of macroeconomic tail risks. The natural candidate for such a measure is the likelihood that log value-added (or output) falls below a certain threshold, that is, $\mathbb{P}(y<\mathbb{E} y-c)$ for some constant $c>0$. Clearly, for a given $c$, a larger $\mathbb{P}(y<\mathbb{E} y-c)$ means that the economy exhibits large negative deviations from its trend at a higher frequency. We can thus define the following concept:

Definition 2. The economy does not exhibit macroeconomic tail risks if for any $c>0$, there exists $\lambda>0$ such that

$$
\mathbb{P}(y<\mathbb{E} y-c) \leq e^{-\lambda n+o(n)} .
$$

In other words, the economy exhibits no macroeconomic tail risks if large economic downturns that may arise due to microeconomic shocks are exponentially unlikely. Consequently, in any such economy, idiosyncratic microeconomic shocks cannot lead to large output contractions. Conversely, we say the economy exhibits macroeconomic tail risks if for some $c>0$, the above inequality holds for no $\lambda>0$.

Finally, we define the notion of normally distributed aggregate fluctuations in the usual fashion:

\footnotetext{
${ }^{8}$ Given two sequences of positive numbers $\left\{a_{n}\right\}$ and $\left\{b_{n}\right\}$, we write $a_{n} \sim b_{n}$ if the two sequences grow with $n$ at the same rate. On the other hand, we write $a_{n}=o\left(b_{n}\right)$ if $a_{n} / b_{n} \rightarrow 0$ as $n \rightarrow \infty$.

${ }^{9}$ Acemoglu et al. (2012) study the behavior of output volatility in a multi-sector economy and show that the presence of input-output linkages can transform idiosyncratic firm- or sector-level shocks to non-trivial fluctuations at the aggregate level. They show that high variability in the sectors' roles as suppliers of intermediate goods leads to a higher volatility at the aggregate level.
} 
Definition 3. Aggregate fluctuations are normally distributed if

$$
y / \sigma_{y} \stackrel{d}{\longrightarrow} \mathcal{N}(0,1)
$$

Hence, in any such economy, the normal distribution provides a reasonable approximation to the nature of GDP fluctuations.

The previous three definitions introduce three separate, even if related, measures of aggregate fluctuations, corresponding, respectively, to the standard deviation of GDP, the presence of macroeconomic tail risks, and the shape of the distribution of GDP. We will next establish that depending on the pattern of input-output linkages and the nature of microeconomic shocks, macroeconomic tail risks may coexist with a distribution that is normal away from the tails (and that may even have a relatively small standard deviation), thus providing a possible explanation for the patterns of fluctuations observed in Figure 1.

\subsection{Balanced Economies: Where No Tail Risks Arise}

In order to develop the link between the nature of macroeconomic tail risks and the input-output linkages within the economy, we first study the class of balanced economies as a benchmark. These are economies in which all sectors play roughly symmetric roles as input suppliers to the rest of the economy. More formally:

Definition 4. An economy is balanced if the share of each sector's output in the input supply of the rest of the economy is uniformly bounded from above; that is, there exists $\bar{v}$ such that $\max _{i} v_{i} \leq \bar{v}$, regardless of the level of disaggregation $n$.

In other words, in a balanced economy, there is a limit to the extent of asymmetry in the roles of different sectors as input suppliers. Figure 2(a) depicts one such economy, in which each sector is an input supplier to a single other sector. Balanced economies lie at the opposite end of the spectrum from the economy depicted in Figure 2(b), where one sector is the sole supplier of all other sectors. We have the following result:

Theorem 1. Suppose that the economy is balanced. Then,

(a) The economy's aggregate volatility is trivial.

(b) The economy exhibits no macroeconomic tail risks.

(c) Aggregate fluctuations are normally distributed.

This theorem extends and strengthens the results of Acemoglu et al. (2012) by showing that in any balanced economy, intersectoral input-output linkages play no significant role in creating macroeconomic fluctuations: not only microeconomic shocks to disaggregated sectors cannot lead 


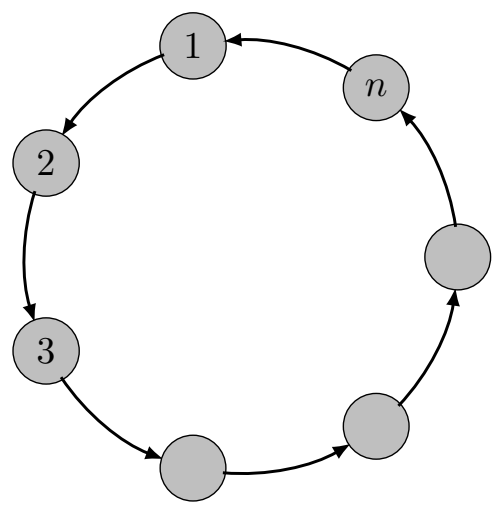

(a) An example of a balanced economy in which all industries take identical roles as input suppliers to other sectors.

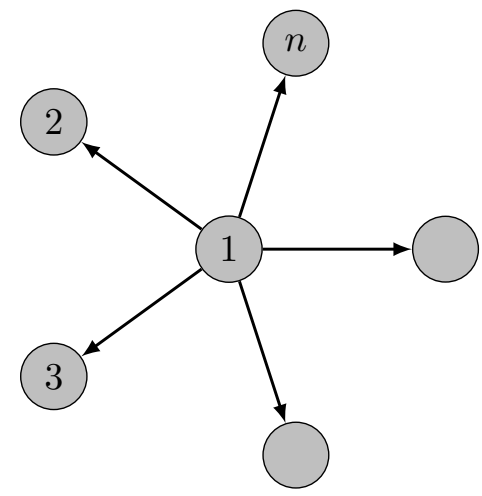

(b) An example of an unbalanced economy in which one sector is the sole supplier of all other sectors within the economy

Figure 2. Network representations of (a) a balanced economy and (b) an unbalanced economy.

to volatility at the aggregate level, but also large economic downturns are extremely unlikely. Furthermore, the normal distribution provides a reasonable approximation to the nature of GDP fluctuations. In this sense, Theorem 1 highlights that as long as there is little asymmetry in the sectors' roles as input suppliers, the economy's aggregate volatility would function as a sufficient statistic for the behavior of macroeconomic variables. The other key observation is that Theorem 1 holds, regardless of the distribution of microeconomic shocks: no matter how shocks are distributed, their impact on the nature of GDP fluctuations are negligible.

Theorem 1 thus essentially provides a general "irrelevance result" for balanced economies, highlighting that idiosyncratic shocks to disaggregated sectors have no significant aggregate effects (measured in terms of output volatility, distribution, or the likelihood of large downturns). Moreover, it provides a justification for the usual practice of (i) relying on output variance as a reasonable proxy for the nature of aggregate fluctuations; and (ii) approximating the deviations of aggregate economic variables from their trends with a normal distribution.

In the remainder of the paper, we show that such practices may no longer be justified if the economy's input-output linkages are not balanced. We demonstrate that in general, depending on the shape of the microeconomic shocks' distribution, the equivalence between trivial volatility, absence of macroeconomic tail risks and normally distributed fluctuations may break down.

\subsection{Normal Shocks}

We first focus on the case in which microeconomic shocks are normally distributed. The normal distribution serves as an analytically tractable example of a distribution with extremely thin, superexponential tails. ${ }^{10}$

\footnotetext{
${ }^{10}$ A distribution is said to have a super-exponential tail if it satisfies $\lim _{t \rightarrow \infty} \log \bar{F}(t) / t=-\infty$, where $\bar{F}(t)=1-F(t)$ is the corresponding counter-cumulative distribution function.
} 
Theorem 2. Suppose that microeconomic shocks are normally distributed. Then,

(a) Aggregate fluctuations are normally distributed.

(b) Aggregate volatility is a sufficient statistic for the likelihood of large downturns.

(c) The economy exhibits no macroeconomic tail risks if and only if its aggregate volatility is trivial.

Thus, in the presence of normally distributed microeconomic shocks, GDP fluctuations can be well-approximated by a normal distribution, even if the economy's input-output linkages are unbalanced. Furthermore, with normal shocks, output variance remains a reasonable proxy for the nature of aggregate fluctuations: economies that exhibit high levels of aggregate volatility are also more likely to experience deeper and more frequent recessions. ${ }^{11}$ In fact, part (c) of the above result shows that as long as the economy's aggregate volatility is trivial, large economic downturns are extremely unlikely.

Our next result provides a comparison of the extent of macroeconomic tail risks across different economies when shocks are normally distributed, and shows that this is related to the vector of the sectoral sales shares, $\left(v_{1}, \ldots, v_{n}\right)$, defined in (2).

Proposition 1. Suppose that microeconomic shocks are normally distributed. Then, an economy with a higher $\sigma_{v}=\operatorname{stdev}\left(v_{1}, \ldots, v_{n}\right)$ exhibits more macroeconomic tail risks.

In other words, as long as microeconomic shocks are normally distributed, the frequency and depth of large economic downturns crucially depend on the variability of different sectors' roles as input suppliers. Thus, for example, deep recessions are significantly more likely in the economy depicted in Figure 2(b) compared to the one depicted in Figure 2(a), in which all sectors take identical roles as input supplier. In fact, given that in any balanced economy $\sigma_{v} \leq \bar{v} / 2$ regardless of the number of sectors $n$, an immediate corollary to the above result implies that balanced economies exhibit no macroeconomic tail risks, an observation consistent with Theorem $1 .{ }^{12}$

However, Proposition 1 also implies that the converse of Theorem 1 is not true in general: there are unbalanced economies that exhibit no macroeconomic tail risks in the presence of normally distributed shocks. This can be seen by considering the stylized economy depicted in Figure 3, in which sector 1 is the sole supplier to $k_{n}$ many sectors, and the output of the rest of the sectors are not used as intermediate goods for production. It is immediate that such an economy is not balanced. Nevertheless, one can also verify that as long as $k_{n} / n \rightarrow 0, \sigma_{v}$ is uniformly bounded above by some constant for all values of $n-$ similar to the class of balanced economies. Thus, such an economy

\footnotetext{
${ }^{11}$ Note that statement (b) of Theorem 2 is not an immediate corollary of statement (a). In fact, as we will show in Subsection 3.3, in the presence of non-normal shocks, aggregate volatility may no longer serve as a sufficient statistic for the extent of macroeconomic tail risks even when aggregate fluctuations are normally distributed (in the sense of Definition 3).

${ }^{12}$ Recall that by definition, an economy is balanced if the sales shares of all sectors are uniformly upper bounded by some $\bar{v}$ for all values of $n$.
} 


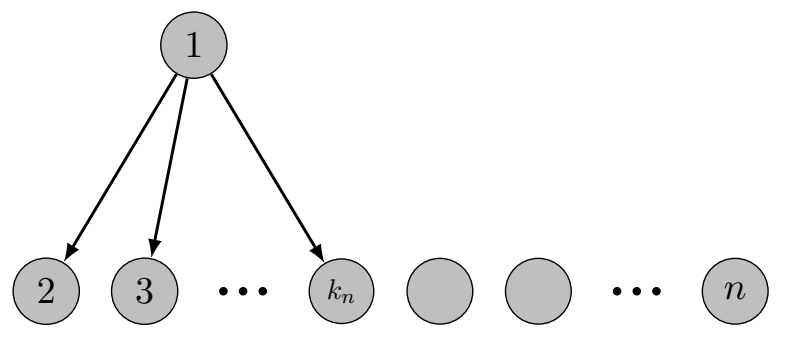

Figure 3. An economy in which sector 1 is the input supplier to $k_{n}$ many sectors.

exhibits no macroeconomic tail risks when microeconomic shocks are normally distributed, despite the fact that it is unbalanced.

Combining this observation with Theorem 2 implies that there exists a fairly large class of unbalanced economies such that in the presence of normally distributed microeconomic shocks, they exhibit no tail risks, have trivial aggregate volatility, and have normally distributed aggregate fluctuations. Consequently, for sizable fluctuations to arise, either (i) input-output linkages within the economy have to be extremely unbalanced (for example, as in the economy depicted in Figure 2(b)), a property that as we will show in Section 5, the US input-output matrix does not seem to satisfy; or as we show next, (ii) microeconomic shocks need to have thicker tails than the normal distribution.

As a final remark, we note that in view of Theorem 2(b), $\sigma_{v}$ also serves as a sufficient statistic for the economy's aggregate volatility. This is in line with the results of Acemoglu et al. (2012), that higher asymmetry in different sectors' role as input suppliers would lead to larger volatility at the aggregate level.

\subsection{Exponential-Tailed Shocks}

We next consider the case in which the tail probabilities of microeconomic shocks decay exponentially fast. More formally:

Definition 5. Microeconomic shocks have exponential tails if

$$
\lim _{t \rightarrow \infty} \frac{1}{t} \log \bar{F}(t)=-\gamma
$$

for some constant $\gamma>0$, where $\bar{F}$ is the common, counter-cumulative distribution of microeconomic shocks $\epsilon_{i}$.

For example, microeconomic shocks exhibit exponential tails if $\bar{F}(t)=L(t) e^{-\gamma t}$ for some polynomial function $L(t) .{ }^{13}$ Clearly, distributions belonging to this class have slightly heavier tails than the normal distribution (but are nowhere as heavy-tailed as the Pareto distribution). Our next theorem provides a sharp converse to Theorem 1(b) for exponential-tail shocks:

\footnotetext{
${ }^{13}$ The Laplace, gamma and hyperbolic distributions belong to this class.
} 
Theorem 3. Suppose that microeconomic shocks have exponential tails. The economy exhibits no macroeconomic tail risks if and only if it is balanced.

Therefore, in the presence of exponential-tailed shocks, any unbalanced economy exhibits macroeconomic tail risks. More importantly, contrasting Theorem 3 with the discussion following Proposition 1 also implies that there exists a fairly large class of unbalanced economies, such as the one depicted in Figure 3, that exhibit significant macroeconomic tail risks in the presence of exponentiallytailed shocks, even though the same economies exhibit no tail risks when shocks are normally distributed. In other words, replacing normally distributed microeconomic shocks with exponential shocks - which have only slightly thicker tails - would dramatically increase the likelihood of large economic downturns. Notably, this is despite the fact that the presence of exponentially distributed shocks has no impact on the standard deviation of GDP or the shape of its distribution away from the tails. Furthermore, this observation illustrates that the "irrelevance result" highlighted in Subsection 3.1 does not hold if input-output linkages in the economy are unbalanced. Rather, in contrast to the class of balanced economies, the shape and nature of microeconomic shocks play a first-order role in shaping the likelihood and depth of large economic downturns.

Our next result provides a comparison of the extent of macroeconomic tail risks across different economies when sectors are subject to exponential-tailed shocks.

Proposition 2. Suppose that microeconomic shocks have exponential tails. Then, an economy with a higher $v_{\max }=\max \left(v_{1}, \ldots, v_{n}\right)$ exhibits more macroeconomic tail risks.

This result highlights yet another aspect of how exponentially-tailed shocks affect the likelihood of large economic downturns differently from normally distributed ones. More specifically, comparing Propositions 1 and 2 illustrates that, unlike the case where shocks are normally distributed, the extent of macroeconomic tail risks in the presence of exponential-tailed shocks depends on the sales share of the most important supplier in the economy. This is in fact the reason why the economy depicted in Figure 3 is observationally equivalent to a balanced economy when shocks are normally distributed, but behaves considerably differently when they are exponentially distributed.

Finally, the next proposition shows that, in the presence of exponential-tailed shocks, macroeconomic tail risks can arise even when aggregate volatility is trivial and GDP is normally distributed:

Proposition 3. Suppose that microeconomic shocks have exponential tails. Then, there exists a class of economies with trivial aggregate volatility and normally distributed aggregate fluctuations that exhibits macroeconomic tail risks.

The significance of Proposition 3 is twofold. First, it shows that if the economy's input-output linkages are unbalanced and microeconomic shocks have slightly thicker tails than a normal distribution, then aggregate volatility is no longer a sufficient statistic for the likelihood of large eco- 
nomic downturns, as the economy may exhibit frequent output contractions. Once again, this is even though aggregate volatility is still trivial. ${ }^{14}$

More importantly, however, Proposition 3 highlights how the presence of input-output linkages can result in patterns similar to the ones documented in Figure 1. In particular, it illustrates that the propagation of microeconomic shocks - as long as they are not extremely thin-tailed — through the input-output linkages can concentrate risks at the tails, despite the fact that aggregate output can be well-approximated by a normal distribution away from the tails. In fact, our quantitative exercise in Section 5 suggests that the US input-output matrix belongs to the class of economies identified in Proposition 3.

\section{Micro vs. Macro Shocks}

In this section, we further clarify how micro shocks, propagated through input-output linkages within the economy, play a role similar to macro shocks (or to micro shocks that are much larger). To this end, we first establish an equivalence result between an unbalanced economy subject to "thin-tailed" microeconomic shocks (as we have assumed so far) and an economy that is subject to "heavy-tailed" shocks (where the likelihood of extreme shocks is relatively high as in the Pareto distribution). The reason why we refer to such impulses as "macro shocks" is that under heavy-tailed distributions, a shock to a single sector or firm that will, by itself (and without any propagation or amplification), move the entire GDP is non-trivial. Our next main result in this section is that inputoutput linkages not only lead to macroeconomic tail risks, but can also generates tail comovement (i.e., simultaneous large falls in the output of many sectors), akin to the effects of aggregate shocks.

\subsection{Heavy-Tailed Shocks}

Our attention so far was restricted to normal and exponential-tailed shock distributions, all of which belong to the class of thin-tailed probability distributions - where the likelihood of large microeconomic shocks is small. In this subsection, we show that input-output linkages may translate such thin-tailed idiosyncratic shocks into aggregate effects that can only arise due to heavy-tailed shocks in the absence of input-output linkages. In other words, we show that the presence of intersectoral input-output linkages in the economy has the same effect on the size of macroeconomic tail risks as subjecting the firms to shocks with heavier tails. This equivalence result is interesting in part because it clarifies how input-output linkages magnify the effects of relatively small shocks.

To develop this point, we focus on the likelihood of large economic downturns in balanced economies, but unlike our earlier results, we now assume that microeconomic shocks are heavytailed, in particular, drawn from a stable distribution with parameter $\alpha \in(1,2)$, which exhibits

\footnotetext{
${ }^{14}$ As stated, Proposition 3 is a "possibility result," rather than a characterization result. A complete characterization of the class of economies with such properties is provided in the Appendix, as it requires more notation.
} 
Pareto tails. In particular, ${ }^{15}$

$$
\lim _{t \rightarrow \infty} \frac{1}{t} \log \bar{F}(t)=-\alpha
$$

Note that a smaller $\alpha$ corresponds to a distribution with heavier tails, according to which large shocks are more likely. We have the following result:

Proposition 4. For any balanced economy with heavy-tailed microeconomics shocks, there exists an unbalanced economy with exponential-tailed shocks that exhibits identical macroeconomic tail risks.

The above result thus underscores that large economic downturns may occur regularly not necessarily due to (aggregate or idiosyncratic) shocks that are drawn from heavy-tailed distributions, but rather as a consequence of the interplay of relatively thin-tailed distributions with the economy's input-output linkages. Put differently, input-output linkages can fundamentally reshape the distribution of aggregate output by concentrating risk at the tails, thus increasing the likelihood of large economic downturns from infinitesimal to substantial. This observational equivalence result thus provides a novel solution to what Bernanke et al. (1996) refer to as the "small shocks, large cycles puzzle" by arguing that the interaction between the economy's input-output linkages and the shape of the microeconomic shocks' distribution may lead to sizable fluctuations at the aggregate level.

\subsection{Tail Comovements}

So far, we quantified the extent of macroeconomic tail risks by the likelihood that log value-added (or output) falls below a certain threshold. Alternatively, one can quantify such tail risks by measuring the likelihood that the output of a significant fraction of sectors simultaneously fall below their respective means by a certain amount:

Definition 6. The economy does not exhibit tail comovement if for any $c>0$ and $\xi<1$, and for any subset $B$ of sectors of size $\xi n$, there exists $\lambda>0$ such that

$$
\mathbb{P}\left(\hat{y}_{i}<\mathbb{E} \hat{y}_{i}-c, \forall i \in B\right) \leq e^{-\lambda n+o(n)},
$$

where $\hat{y}_{i}=\log \left(y_{i}\right)$ is the logarithm of output of sector $i$.

In other words, the economy exhibits no tail comovement if large, simultaneous output drops in a significant number of sectors are exponentially unlikely.

Our next result shows that input-output linkages not only translate idiosyncratic microeconomic shocks into macroeconomic tail risks in the sense of Definition 2, but can also create tail comovement. Since characterizing joint tail probabilities for a large number of sectors is somewhat more

\footnotetext{
${ }^{15}$ Stable distributions with parameter $\alpha<2$ belong to the class of distributions with sub-exponential tails, in the sense that $\lim _{t \rightarrow \infty} \overline{F * F}(t) / \bar{F}(t)=2$. For more on stable and sub-exponential distributions, see Zolotarev (1986).
} 
involved, we derive the results in this subsection for a special case of our economy. Namely, we assume that the input-output matrix $W$ can be written as $W=(1-\beta) \tilde{W}$, where $\tilde{W}$ is independent of $\beta$. We will then consider the limit where $\beta \rightarrow 0$, so that the role of intermediate inputs in the production function (1) becomes arbitrarily close to 1 . At the same time, in order for the sales shares $v_{i}$ to remain well-defined, we rescale these values by defining $u_{i}=\lim _{\beta \rightarrow 0} \beta v_{i}$. This limiting regime reduces the non-linearity in sectoral interactions, enabling us to derive sharper results:

Proposition 5. Suppose that the economy's underlying input-output network is connected. As $\beta \rightarrow 0$, the economy exhibits tail comovement if and only if it exhibits macroeconomic tail risks.

Proposition 5 implies that all our earlier results for the emergence of macroeconomic tail risks from microeconomic shocks generalize to tail comovements. For example, using our earlier results we can obtain a characterization for the extent of tail comovements in the presence of normal and exponentially-tailed microeconomic shocks.

Corollary 1. Suppose that microeconomic shocks are normally distributed. Then, as $\beta \rightarrow 0$, an economy with a higher $\sigma_{u}=\operatorname{stdev}\left(u_{1}, \ldots, u_{n}\right)$ exhibits greater tail comovement. On the other hand, when microeconomic shocks have exponential tails, an economy with a higher $u_{\max }=\max \left\{u_{1}, \ldots, u_{n}\right\}$ exhibits greater tail comovement.

More importantly, however, Proposition 5 shows that input-output linkages can translate idiosyncratic shocks to large simultaneous output drops across a large number of sectors, as if they were all hit by the same aggregate shock. It is worth noting that this result sharply contrasts with those for economies without input-output linkages, such as Gabaix (2011), in which aggregate fluctuations may arise from firm-level shocks when firm-size distribution is extremely heavy-tailed: even though such an economy may be observationally equivalent to an economy with input-output linkages at the aggregate level, the two exhibit significantly different behavior in terms of tail comovements. To see this divergence between the two approaches, consider an economy in the spirit of Gabaix (2011), in which the $\log$ output of sector $i$ is given by $\hat{y}_{i}=s_{i} \epsilon_{i}$, where $s_{i}$ captures the size of sector $i$ and $\epsilon_{i}$ is the corresponding microeconomic shock. In such an economy, the likelihood that the log-output of $\xi n$ many sectors fall by an amount $c$ below their respective means is given by $\prod_{i=1}^{\xi n} \bar{F}\left(c / s_{i}\right)$, which is smaller than $(1 / 2)^{\xi n}$. Therefore, in any such economy, tail comovements is exponentially unlikely regardless of firm-size distribution and the distribution of microeconomic shocks, in contrast to the prediction of Proposition 5 for input-output economies.

\section{A Simple Quantitative Illustration}

In this section, we provide a simple quantitative exercise to highlight the role of input-output linkages and the nature of microeconomic shocks in the emergence of macroeconomic tail risks. Using 


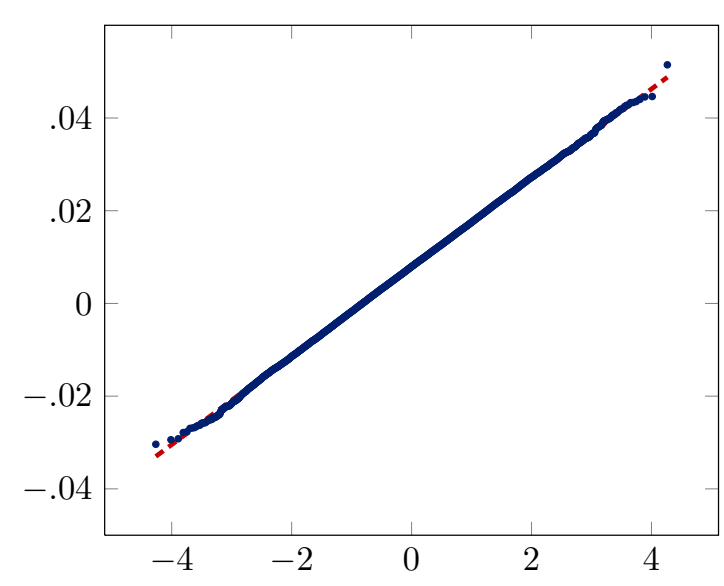

(a) normally distributed shocks

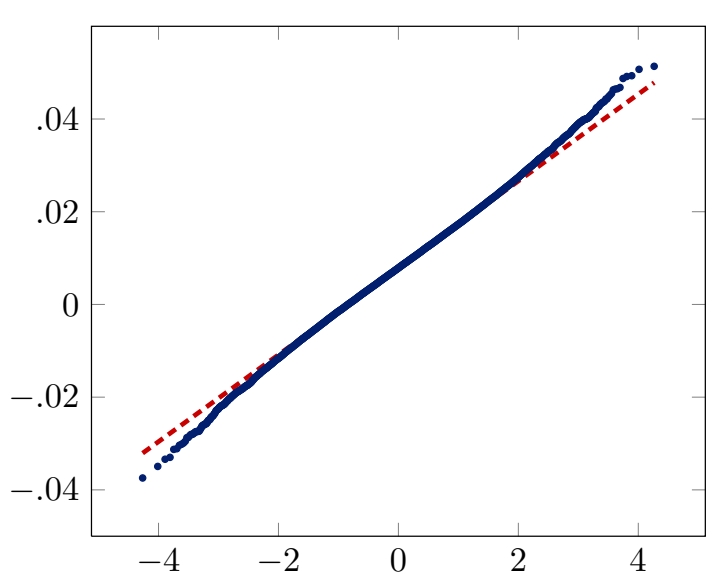

(b) exponentially distributed shocks

Figure 4. Q-Q plots for the model with the input-output matrix given by the 2002 US commodity-by-commodity direct requirements table. Panels (a) and (b) depict the Q-Q plot when microeconomic shocks are drawn, respectively, from a normal distribution and a symmetric exponential distribution.

the input-output table of the US economy, we show that when sectoral shocks are normally distributed, the economy exhibits no macroeconomic tail risks, whereas such tail risks emerge when shocks are exponentially distributed. Also, consistent with our theoretical results, we show that had the US input-output matrix been balanced, no such macroeconomic tail risks would have emerged.

For the purpose of this exercise, we use the 2002 commodity-by-commodity direct requirements table, compiled by the Bureau of Economic Analysis. The typical $(i, j)$ entry of this table captures the value of spending on commodity $i$ per dollar of production of commodity $j \cdot{ }^{16}$ Given our assumption of Cobb-Douglas sectoral production functions, this entry is proportional to the corresponding entry in the input-output matrix, $w_{i j}$ in (1). We can thus construct the economy's Leontief matrix $L$ and the sales share of each sector (defined in (2)). In this exercise, we set $\beta=0.4$, which corresponds to one minus the median of the value of $\sum_{j=1}^{n} w_{i j}$ in the 2002 direct requirements table.

We first study the distribution of aggregate output when microeconomic (sectoral) shocks are drawn from a normal distribution. The mean and variance of the shocks are chosen to make the first two moments of the simulated economy's aggregate output match the first two moments of the US postwar GDP growth rate. The corresponding Q-Q plot of the aggregate output is depicted in Figure 4(a). ${ }^{17}$ The close correspondence between the normal distribution, shown again by the dashed red line, and the distribution of aggregate output confirms our theoretical results - that when microeconomic shocks are normal, the normal distribution provides a fairly good approximation for the distribution of aggregate output throughout. In particular, in contrast to the pattern documented in Figure 1, there are no significant deviations at the tail. We also performed the Anderson-Darling and Jarque-Bera tests of normality, with the resulting test statistics given by 0.36 and 1.27, respectively.

\footnotetext{
${ }^{16}$ Some basic descriptive statistics about these input-output linkages is given in Acemoglu et al. (2012).

${ }^{17}$ We take 50,000 draws from the implied distributions to construct these figures and the test statistics.
} 


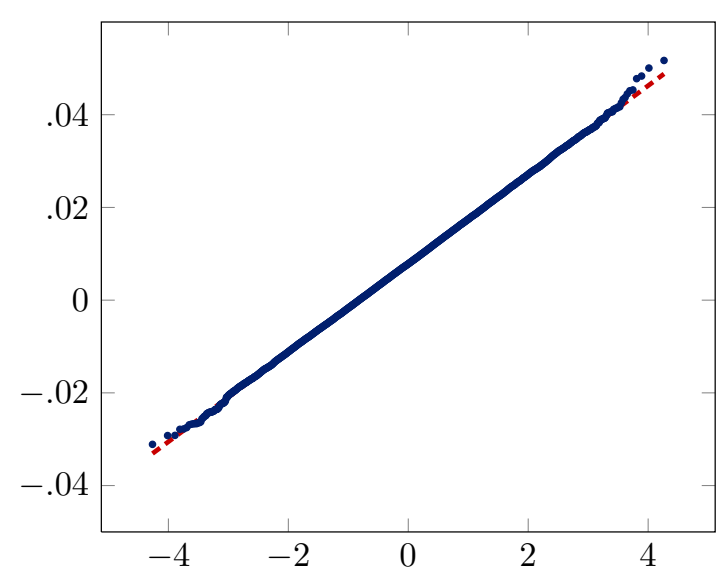

Figure 5. The Q-Q plot for aggregate output versus the normal distribution for a balanced economy subject to exponentially distributed microeconomic shocks.

In neither case can we reject normality at the $15 \%$ significance level.

The pattern is very different, however, when microeconomic shocks are drawn from a symmetric exponential distribution. Figure 4(b) depicts the corresponding Q-Q plot. Away from the tails, the distribution is again well approximated by the dashed red line corresponding to the normal distribution. However, and in contrast to the pattern observed in Figure 4(a), there are now systematic deviations from the normal distribution at the tails - corresponding to significant macroeconomic tail risks. The Anderson-Darling and Jarque-Bera tests of normality yield test statistics of 6.22 and 157.76, respectively, rejecting normality at the $1 \%$ level in both cases. This overall pattern confirms the theoretical results from Section 3, in particular, that microeconomic shocks from the distribution with slightly thicker tails combined with unbalanced input-output linkages can lead to a substantial increase in the likelihood of large economic downturns. ${ }^{18}$

It is important to note that the unbalanced nature of input-output linkages in the US economy is key for obtaining the deviations from normality observed in Figure 4(b). In particular, recall from Theorem 1 that had we used a balanced input-output matrix instead of the fairly unbalanced US input-output matrix, there would be no significant macroeconomic tail risks. In fact, performing the above quantitative exercise for a balanced economy of the same size, $n=417$ (for example, one in which all sectors depend equally on inputs from all other sectors) with exponentially distributed microeconomic shocks, we obtain the pattern depicted in Figure 5. As the figure suggests, the distribution of aggregate output is very close to a normal distribution. The Anderson-Darling and Jarque-Bera test statistics are now 0.40 and 2.56, neither of which is significant at the $15 \%$ level.

Overall, this simple quantitative exercise establishes that (i) the US input-output matrix is sufficiently unbalanced to lead to potentially significant macroeconomic tail risks in the presence of

\footnotetext{
${ }^{18}$ Similar results are obtained when we allow $\sum_{j=1}^{n} w_{i j}$ to vary across sectors. In this case, the Anderson-Darling and Jarque-Bera test statistics of normality are, respectively, 0.29 and 0.99 when microeconomic shocks are normally distributed (neither of which are significant at the $15 \%$ level), and 1.94 and 43.13 when microeconomic shocks are exponentially distributed (thus rejecting normality at $10 \%$ and $0.1 \%$, respectively).
} 
exponentially-distributed microeconomic shocks; and (ii) it is indeed the interplay of this unbalanced input-output structure and the shape of the distribution of microeconomic shocks that is key for generating such tail risks.

\section{Conclusions}

Even though the normal distribution, widely used in modern macroeconomics, is generally a good approximation to the nature of aggregate fluctuations, it severely underpredicts the frequency of large economic downturns. This observation calls for theoretical models that can simultaneously generate (i) significant macroeconomic tail risks and (ii) approximately normally distributed fluctuations away from the tails. Our main contribution is to provide such a model.

Our model relies on the propagation of (idiosyncratic) microeconomic shocks over the inputoutput linkages across different firms or sectors within the economy. We show that forces related to the central limit theorem ensure that the distribution of aggregate output is close to normal especially away from the tails. But crucially, depending on the nature of input-output linkages and the shape of the distribution of these microeconomic shocks, significant tail risks can also emerge. In particular, when microeconomic shocks have an exponential distribution (rather than the more thin-tailed normal distribution) and when the input-output structure is unbalanced (meaning that some sectors play a more important role as input suppliers to the rest of the economy than others), the economy exhibits significant macroeconomic tail risks. In particular, an unbalanced economy subject to thin-tailed microeconomic shocks generates large economic downturns as frequently as an economy subject to heavy-tailed shocks but with a balanced input-output structure. We then systematically characterize the nature of tail risks in such economies and show how the same forces that lead to tail risks in aggregate output can also create "tail comovements," whereby the outputs of many sectors may drop simultaneously, as if the economy is subject to an aggregate shock.

In summary, our results highlight the importance of separately studying the determinants of large economic downturns, since such macroeconomic tail risks may vary significantly even across economies that exhibit otherwise identical behavior for moderate deviations.

We view our paper as a first step in a systematic investigation of the causes of large economic downturns, which have attracted comparatively little attention so far. A particularly important area for future work is to combine input-output or other linkages that are capable of generating large economic downturns from microeconomic shocks with richer microeconomic shocks (rather than the simple productivity shocks considered here) as well as richer microeconomic structures (rather than the perfectly competitive economy studied here). Another equally important area is of course the empirical study of the nature and causes of large economic downturns, and whether they support economic mechanisms built on the propagation of microeconomic shocks through input-output linkages or other microeconomic interactions across sectors and firms. 


\section{A Proofs}

We start by stating two propositions, the proofs of which can be found in Acemoglu et al. (2012). The first proposition characterizes the economy's aggregate output in terms of microeconomic shocks and sales shares defined in (2). The second proposition provides conditions for normally distributed aggregate fluctuations in terms of the economy's sales share vector.

Proposition A.1. Let y denote the economy's log value added. Then, $y=(\beta / n) \sum_{i=1}^{n} v_{i} \epsilon_{i}$.

Proposition A.2. Suppose that microeconomic shocks are not normally distributed. Let $\|v\|=\sqrt{\sum_{i=1}^{n} v_{i}^{2}}$ and $v_{\max }=\max \left(v_{1}, \ldots, v_{n}\right)$. Aggregate fluctuations are normally distributed if only if $v_{\max } /\|v\| \rightarrow 0$ as $n$ increases.

\section{Proof of Theorem 1}

Proof of part (a) By Proposition A.1 it is immediate that

$$
\sigma_{y}^{2}=\frac{\beta^{2} \sigma^{2}}{n^{2}} \sum_{i=1}^{n} v_{i}^{2}
$$

Furthermore, (3) implies that $v_{i} \geq 1$ for all sectors $i$. Therefore,

$$
\sigma_{y}^{2} \geq \beta^{2} \sigma^{2} / n
$$

On the other hand, by definition, in any balanced economy, $v_{i} \leq \bar{v}$ for all sectors $i$ and regardless of the level of disaggregation. Therefore,

$$
\sigma_{y}^{2} \leq \frac{\beta^{2} \sigma^{2} \bar{v}}{n^{2}} \sum_{i=1}^{n} v_{i}=\bar{v} \beta \sigma^{2} / n
$$

where we are using the fact that $\sum_{i=1}^{n} v_{i}=n / \beta$. Combining inequalities (5) and (6) then implies $\sigma_{y} \sim 1 / \sqrt{n}$.

Proof of part (b) Proposition A.1 implies that if $\epsilon_{i}<-c$ for all sectors $i$, then $y<-c$. Therefore,

$$
[F(-c)]^{n} \leq \mathbb{P}(y<-c)
$$

and as a result,

$$
\limsup _{n \rightarrow \infty} \frac{1}{n}|\log \mathbb{P}(y<-c)|<\infty .
$$

On the other hand, Chernoff's inequality implies that

$$
\mathbb{P}(y<-c) \leq e^{-n \delta c} \mathbb{E}\left(e^{n \delta y}\right)=e^{-n \delta c} \prod_{i=1}^{n} \mathbb{E}\left(e^{\delta \beta v_{i} \epsilon_{i}}\right),
$$


for any $\delta \geq 0$. Taking logarithms from both sides of the above inequality, we have

$$
\log \mathbb{P}(y<-c) \leq-n \delta c+\sum_{i=1}^{n} g\left(\delta \beta v_{i}\right),
$$

where $g(t)=\log \mathbb{E}\left[\exp \left(t \epsilon_{i}\right)\right]$ is the cumulant-generating function corresponding to random variable $\epsilon_{i}$. Therefore,

$$
\frac{1}{n} \log \mathbb{P}(y<-c) \leq-\delta c+\max _{\delta \beta \leq t \leq \delta \bar{v}} g(t),
$$

where we are using the fact that $1 \leq v_{i} \leq \bar{v}$ for all $i$. Let $\Lambda(\delta)=\delta c-\max _{0 \leq t \leq \delta \beta \bar{v}} g(t)$. It is immediate that $\Lambda(0)=0$ and $(1 / n) \log \mathbb{P}(y<-c) \leq-\Lambda(\delta)$. Furthermore, by the envelope theorem, $\Lambda^{\prime}(0)>0$. Consequently, there exists small enough $\delta>0$ such that $\Lambda(\delta)>0$, and therefore,

$$
\liminf _{n \rightarrow \infty} \frac{1}{n}|\log \mathbb{P}(y<-c)| \geq \Lambda(\delta)>0 .
$$

Combining inequality (8) with (7) thus implies

$$
|\log \mathbb{P}(y<-c)| \sim n
$$

implying that there exists $\lambda>0$ such that $\mathbb{P}(y<-c)=e^{-\lambda n+O(n)}$.

Proof of part (c) Recall that $v_{i} \geq 1$ for all $i$. Therefore, $\|v\| \geq \sqrt{n}$. Therefore, the fact that the economy is balanced implies that $v_{\max } /\|v\| \leq \bar{v} / \sqrt{n}$. Therefore, by Proposition A.2, aggregate fluctuations are normally distributed regardless of the shock distributions.

\section{Proof of Theorem 2}

From Proposition A.1 aggregate output is a linear combination of microeconomic shocks. Since space microeconomic shocks are normally distributed, it is then immediate that $y$ has to be normally distributed for all levels of disaggregation $n$, proving part (a).

Furthermore, the mean and variance of any normally distributed random variable are sufficient statistics for the the whole distribution. Given that $\mathbb{E} y=0$, the standard deviation of aggregate output, $\sigma_{y}$, is a sufficient statistic for the distribution of aggregate output, including the likelihood of large downturns, proving part (b).

Finally, to prove part (c), note that $y$ is normally distributed with mean 0 and standard deviation $\sigma_{y}=\beta \sigma\|v\| / n$. Therefore, the likelihood of large downturns satisfies

$$
\mathbb{P}(y<-c)=1-\Phi\left(c / \sigma_{y}\right)
$$

where $\Phi$ is the standard normal cumulative probability distribution. On the other hand, it is wellknown that

$$
\lim _{t \rightarrow \infty} \frac{t[1-\Phi(t)]}{\phi(t)}=1
$$


where $\phi$ denotes the standard normal probability density function. ${ }^{19}$

$$
\mathbb{P}(y<-c) \sim \frac{\phi\left(c / \sigma_{y}\right)}{c / \sigma_{y}}=\frac{\sigma_{y}}{c \sqrt{2 \pi}} \exp \left(-\frac{c^{2}}{2 \sigma_{y}^{2}}\right) .
$$

The right-hand side of the above equality decays to zero exponentially fast, if and only if $\sigma_{y} \sim 1 / \sqrt{n}$. Thus, the economy exhibits tails risks if and only if its aggregate volatility is non-trivial.

\section{Proof of Proposition 1}

Recall from the proof of Theorem 2 that in the presence of normally distributed microeconomic shocks, the likelihood of tail risks satisfies (9). Taking logarithms from both sides implies

$$
|\log \mathbb{P}(y<-c)| \sim 1 / \sigma_{y}^{2}
$$

On the other hand, recall from the proof of Theorem 1 that $\sigma_{y}^{2} \sim\left(1 / n^{2}\right) \sum_{i=1}^{n} v_{i}^{2}$. Furthermore,

$$
\frac{1}{n^{2}} \sum_{i=1}^{n} v_{i}^{2}=\left(\sigma_{v} / n\right)^{2}+1 / \beta^{2},
$$

where we are using the fact that $\sum_{i=1}^{n} v_{i}=n / \beta$. Therefore, increasing $\sigma_{v}$ increases $\mathbb{P}(y<-c)$.

\section{Proof of Proposition 2}

We prove this result by showing that if microeconomic shocks have exponential tails, then

$$
|\log \mathbb{P}(y<-c)| \sim n / v_{\max },
$$

thus establishing that the extent of macroeconomic tail risk in the economy increases in $v_{\max }$.

First, note that (10) hold trivially if $v_{\max } \sim n$. This is due to the fact that whenever $v_{\max } \sim n$, equation (4) guarantees that $\sigma_{y}^{2}>0$, implying that the event $\{y<-c\}$ occurs with some positive probability, regardless of the level of disaggregation. Thus, for the remainder of the proof, we assume that $v_{\max }=o(n)$.

Next, we show that $\lim \sup _{n \rightarrow \infty}\left(v_{\max } / n\right)|\log \mathbb{P}(y<-c)|<\infty$. Note that if $v_{i} \epsilon_{i}<-c n / \beta$ and $\sum_{j \neq i} v_{j} \epsilon_{j}<0$ hold for some $i$, then $y<-c$. Therefore, given the assumption that microeconomic shocks are independent with symmetric distributions, we have

$$
\begin{aligned}
\mathbb{P}(y<-c) & \geq \frac{1}{2} \mathbb{P}\left(v_{\max } \epsilon_{i}<-c n / \beta\right) \\
& =\frac{1}{2} \bar{F}\left(\frac{c n}{\beta v_{\max }}\right)
\end{aligned}
$$

which implies

$$
\limsup _{n \rightarrow \infty}\left(\frac{v_{\max }}{n}\right)|\log \mathbb{P}(y<-c)| \leq \limsup _{n \rightarrow \infty}\left(\frac{v_{\max }}{n}\right)\left|\log \bar{F}\left(\frac{c n}{\beta v_{\max }}\right)\right| .
$$

\footnotetext{
${ }^{19}$ For a proof, see for example, Grimmett and Stirzaker (2001, p. 98). Also note that we can use this equality only if $\sigma_{y} \rightarrow 0$. However, if $\sigma_{y}$ does not decay to zero as the level of disaggregation is increased, then the result holds trivially.
} 
Given that $v_{\max }=o(n)$ and the assumption that microeconomic shocks have exponential tails, the right-hand side of the above inequality is finite. Consequently,

$$
\limsup _{n \rightarrow \infty}\left(\frac{v_{\max }}{n}\right)|\log \mathbb{P}(y<-c)|<\infty
$$

Next, we show that $\liminf _{n \rightarrow \infty}\left(v_{\max } / n\right)|\log \mathbb{P}(y<-c)|>0 .{ }^{20}$ Note that since $F$ has an exponential tail, there exists a strictly positive constant $\gamma$ such that

$$
1-F(t)<e^{-\gamma t}
$$

for all $t>0$. This is due to the fact that the function $-(1 / t) \log [1-F(t)]$ is always positive for $t>0$ and has a strictly positive limit inferior.

On the other hand, given that $F$ is symmetric, we have

$$
\frac{1}{2} \mathbb{E}\left|\epsilon_{i}\right|^{k}=\int_{0}^{\infty} t^{k} d F(t)=\int_{0}^{\infty} k t^{k-1}(1-F(t)) d t
$$

for $k \geq 2$, where we have used integration by parts and the fact that

$$
0 \leq \lim _{t \rightarrow \infty} t^{k}(1-F(t))=\lim _{t \rightarrow \infty} \exp [k \log (t)+\log (1-F(t))]=0 ;
$$

a consequence of the exponential tails assumption. Thus, by (12), there exists a positive constant $r=1 / \gamma$ such that

$$
\frac{1}{2} \mathbb{E}\left|\epsilon_{i}\right|^{k} \leq \int_{0}^{\infty} k t^{k-1} e^{-t / r} d t=r^{k} k !
$$

for all $k \geq 2$. Therefore, for $\delta<n /\left(r \beta v_{\max }\right)$ and for all $i$, we have

$$
\begin{aligned}
\mathbb{E}\left(e^{\delta \beta v_{i} \epsilon_{i} / n}\right) & =1+\sum_{k=2}^{\infty} \frac{\left(\delta \beta v_{i} / n\right)^{k}}{k !} \mathbb{E}\left(\epsilon_{i}^{k}\right) \\
& \leq 1+2 \sum_{k=2}^{\infty}\left(\delta \beta r v_{i} / n\right)^{k} .
\end{aligned}
$$

The above inequality implies that

$$
\mathbb{E}\left(e^{\delta \beta v_{i} \epsilon_{i} / n}\right) \leq 1+\frac{2\left(\delta \beta r v_{i} / n\right)^{2}}{1-\delta \beta r v_{i} / n} \leq \exp \left(\frac{2\left(\delta \beta r v_{i} / n\right)^{2}}{1-\delta \beta r v_{\max } / n}\right),
$$

where we are using the fact that $1+t \leq e^{t}$ for all $t \geq 0$. On the other hand, from Proposition A.1 and Chernoff's inequality, we have

$$
\mathbb{P}(y<-c) \leq e^{-\delta c} \mathbb{E}\left(e^{\delta y}\right)=e^{-\delta c} \prod_{i=1}^{n} \mathbb{E}\left(e^{\delta \beta v_{i} \epsilon_{i} / n}\right) .
$$

Combining the above inequality with (13) implies

$$
\log \mathbb{P}(y<-c) \leq-\delta c+\sum_{i=1}^{n} \frac{2\left(\delta \beta r v_{i} / n\right)^{2}}{1-\delta \beta r v_{\max } / n}=-\delta c+\frac{2(\delta \beta r\|v\| / n)^{2}}{1-\delta \beta r v_{\max } / n}
$$

\footnotetext{
${ }^{20}$ For a similar argument, see, e.g., Teicher (1984).
} 
Letting $\delta=c /\left((2 r \beta\|v\| / n)^{2}+r c \beta v_{\max } / n\right)$ leads to ${ }^{21}$

$$
\begin{aligned}
\log \mathbb{P}(y<-c) & \leq \frac{-c^{2} n^{2}}{8 \beta^{2} r^{2}\|v\|^{2}+2 n \beta r c v_{\max }} \\
& \leq \frac{-n c^{2}}{2 r \beta v_{\max }(4 r+c)}
\end{aligned}
$$

where in the second inequality we are using the fact that $\|v\|^{2} \leq v_{\max } \sum_{i=1}^{n} v_{i}=n v_{\max } / \beta$. Therefore,

$$
\liminf _{n \rightarrow \infty}\left(\frac{v_{\max }}{n}\right)|\log \mathbb{P}(y<-c)| \geq \frac{c^{2}}{8 r^{2} \beta+2 r c \beta}>0 .
$$

Combining inequalities (11) and (14) establishes (10), thus completing the proof.

\section{Proof of Theorem 3}

First, suppose that the economy is balanced. By Theorem 1, such an economy exhibits no macroeconomic tail risks.

To prove the converse, suppose that the economy exhibits no macroeconomic tail risks; i.e., $\mathbb{P}(y<-c) \leq \exp (-\lambda n+o(n))$, which is equivalent to $\liminf _{n \rightarrow \infty}(1 / n) \log |\mathbb{P}(y<-c)|>0$. It is thus immediate that $\lim \sup _{n \rightarrow \infty}(1 / n) \log |\mathbb{P}(y<-c)|>0$. This, however, means that $v_{\max }$ cannot grow with $n$, as otherwise inequality (11) would be violated, implying that the economy has to be balanced.

\section{Proof of Proposition 3}

We prove this result, by first making three observations.

First, recall from (4) that in any economy, $\sigma_{y} \sim\|v\| / n$, where $v$ is the Euclidean norm of vector $v$. Therefore, the economy's aggregate volatility is trivial if and only if

$$
\|v\| \sim \sqrt{n}
$$

Second, Proposition A.2 shows that aggregate fluctuations are normally distributed if $v_{\max } /\|v\| \rightarrow$ 0 as the level of disaggregation $n$ increases. Therefore, if aggregate volatility is trivial, aggregate fluctuations are normally distributed if and only if

$$
v_{\max } / \sqrt{n} \rightarrow 0
$$

Finally, Theorem 3 guarantees that the economy exhibits macroeconomic tail if and only if is unbalanced, which by definition, means that $v_{\max }$ grows with $n$; or put differently,

$$
1 / v_{\max } \rightarrow 0
$$

\footnotetext{
${ }^{21}$ Note that this choice of $\delta$ satisfies $\delta<n /\left(r \beta v_{\max }\right)$, the condition required for deriving (13).
} 
Combining the above three observations implies that in the presence of exponentially-tailed microeconomic shocks, any economy that satisfies (15)-(17) simultaneously, exhibits trivial aggregate volatility, has normally distributed aggregate fluctuations and exhibits tail risks. In fact, these three equations provide a full characterization of the set of economies that belong to this class.

The proof is complete once we show that this set is non-empty. To this end, consider the economy depicted in Figure 3. It easy to verify that if $k_{n}$ is such that $1 / k_{n} \rightarrow 0$ and $k_{n} / \sqrt{n} \rightarrow n$ simultaneously, then the economy satisfies (15)-(17).

\section{Proof of Proposition 4}

Consider a balanced economy that is subject to microeconomic shocks drawn from a stable distribution with parameter $\alpha \in(1,2)$. Recall from Proposition A.1 that the economy's aggregate output is given by $y=(\beta / n) \sum_{i=1}^{n} v_{i} \epsilon_{i}$. Therefore,

$$
\mathbb{P}(y<-c)=\mathbb{P}\left(\sum_{i=1}^{n} v_{i} \epsilon_{i}<-n c / \beta\right) .
$$

On the other hand, it is well-known that for a collection of independent random variables with a common stable distribution of parameter $\alpha$, random variables $\sum_{i=1}^{n} v_{i} \epsilon_{i}$ and $\left(\sum_{i=1}^{n} v_{i}^{\alpha}\right)^{1 / \alpha} \epsilon_{1}$ have identical distributions (see, for example, Zolotarev (1986)). Consequently,

$$
\mathbb{P}(y<-c)=\bar{F}\left(\frac{n c / \beta}{\left(\sum_{i=1}^{n} v_{i}^{\alpha}\right)^{1 / \alpha}}\right) .
$$

Furthermore, recall that in any balanced economy, $v_{i} \sim 1$, thus implying that $\left(\sum_{i=1}^{n} v_{i}^{\alpha}\right)^{1 / \alpha} \sim n^{1 / \alpha}$. Therefore, the fact that microeconomic shocks have a Pareto tail with exponent $\alpha$ implies that in any balanced economy subject to heavy-tail shocks, the extent of macroeconomic tail risks satisfies

$$
\log |\mathbb{P}(y<-c)| \sim n^{1-1 / \alpha}
$$

Comparing the above with (10) shows that the extent of macroeconomic tail risks in the balanced economy above coincides with that of an unbalanced economy that is subject to exponential-tailed shocks for which $v_{\max } \sim n^{1 / \alpha}$.

\section{Proof of Proposition 5}

First, note that the log output of sector $i$ in the competitive equilibrium of the economy can be written as

$$
\hat{y}_{i}=\beta \sum_{j=1}^{n} L_{i j} \epsilon_{j}+\zeta_{i}
$$


where $L$ is the economy's Leontief matrix and $\zeta_{i}$ is some constant that is independent of the the shocks. ${ }^{22}$

On the other hand, for any vector $\tilde{v}$,

$$
\beta L=\beta(I-W)^{-1}=\mathbf{1} \tilde{v}^{\prime}+\beta \sum_{k=0}^{\infty}(1-\beta)^{k}\left(\tilde{W}^{k}-\mathbf{1} \tilde{v}^{\prime}\right) .
$$

If we choose $\tilde{v}$ to be the left eigenvector, corresponding to eigenvalue 1 , of matrix $\tilde{W}$ (that is, $\tilde{v}^{\prime} \tilde{W}=$ $\left.\tilde{v}^{\prime}\right)$, the above equality can be rewritten as

$$
\beta L=\mathbf{1} u^{\prime}+\beta \sum_{k=0}^{\infty}(1-\beta)^{k}\left(\tilde{W}-\mathbf{1} \tilde{v}^{\prime}\right)^{k} .
$$

Given that $\tilde{W}-\mathbf{1} \tilde{v}^{\prime}$ has a spectral radius less than one, the summation on the right hand side converges regardless of the value of $\beta<1$. Therefore, taking the limit of $\beta \rightarrow 0$ implies that the last term vanishes, leading to

$$
\lim _{\beta \rightarrow 0} \beta L=\mathbf{1} \tilde{v}^{\prime}
$$

Combining the above with (18) implies

$$
\lim _{\beta \rightarrow 0} \hat{y}_{i}=\sum_{j=1}^{n} \tilde{v}_{j} \epsilon_{j}+\lim _{\beta \rightarrow 0} \hat{\zeta}_{i} .
$$

Finally, recall that $u_{i}$ is defined as $u_{i}=\lim _{\beta \rightarrow 0} \beta v_{i}$. Therefore, from (19),

$$
u_{i}=\lim _{\beta \rightarrow 0} \sum_{j=1}^{n} \beta L_{j i}=n \tilde{v}_{i}
$$

implying that (20) can be rewritten as

$$
\lim _{\beta \rightarrow 0} \hat{y}_{i}=\frac{1}{n} \sum_{j=1}^{n} u_{j} \epsilon_{j}+\lim _{\beta \rightarrow 0} \hat{\zeta}_{i} .
$$

Thus, as $\beta \rightarrow 0$, the outputs of all sectors become fully correlated with one another regardless of the economy's input-output matrix. Furthermore, by Proposition A.1 sectoral outputs are also fully positively correlated with aggregate output, thus implying that the output of all sectors drop by a fixed level $c$ below their respective means if and only if aggregate output falls below its own mean by c.

\footnotetext{
${ }^{22}$ For a detailed derivation of this result, see Acemoglu et al. (2015).
} 


\section{References}

Acemoglu, Daron, Vasco M. Carvalho, Asuman Ozdaglar, and Alireza Tahbaz-Salehi (2012), “The network origins of aggregate fluctuations.” Econometrica, 80, 1977-2016.

Acemoglu, Daron, Asuman Ozdaglar, and Alireza Tahbaz-Salehi (2010), "Cascades in networks and aggregate volatility.” NBER Working Paper No. 16516.

Acemoglu, Daron, Asuman Ozdaglar, and Alireza Tahbaz-Salehi (2015), "Networks, shocks and systemic risk." In The Oxford Handbook on the Economics of Networks (Yann Bramoullé, Andrea Galeotti, and Brian Rogers, eds.), Oxford University Press.

Atalay, Enghin (2014), “How important are sectoral shocks?” Working paper.

Barro, Robert J. (2006), "Rare disasters and asset markets in the Twentieth century.” The Quarterly Journal of Economics, 121, 823-866.

Bernanke, Ben, Mark Gertler, and Simon Gilchrist (1996), “The financial accelerator and the flight to quality." The Review of Economics and Statistics, 78, 1-15.

Carvalho, Vasco M. (2010), “Aggregate fluctuations and the network structure of intersectoral trade.” Working paper.

Carvalho, Vasco M. and Xavier Gabaix (2013), “The great diversification and its undoing.” American Economic Review, 103, 1697-1727.

Christiano, Lawrence, Martin S. Eichenbaum, and Sergio Rebelo (2011), "When is the government spending multiplier large?" Journal of Political Economy, 119, 78-121.

Christiano, Lawrence J., Martin S. Eichenbaum, and Mathias Trabandt (2015), "Understanding the Great Recession.” American Economic Journal: Macroeconomics.

Cole, Harold L. and Lee E. Ohanian (1999), "The Great Depression in the United States from a Neoclassical perspective.” Federal Reserve Bank of Minneapolis Quarterly Review, 23, 2-24.

Cole, Harold L. and Lee E. Ohanian (2002), "The Great U.K. Depression: A puzzle and possible resolution." Review of Economic Dynamics, 5, 19-44.

di Giovanni, Julian, Andrei A. Levchenko, and Isabelle Méjean (2014), "Firms, destinations, and aggregate fluctuations." Econometrica, 82, 1303-1340.

Dupor, Bill (1999), “Aggregation and irrelevance in multi-sector models.” Journal of Monetary Economics, 43, 391-409. 
Durlauf, Steven N. (1993), “Nonergodic economic growth.” Review of Economic Studies, 60, 349-366.

Eggertsson, Gauti B. and Paul Krugman (2012), "Debt, deleveraging, and the liquidity trap: A fisherminsky-koo approach." Quarterly Journal of Economics, 127, 1469-1513.

Eggertsson, Gauti B. and Neil R. Mehrotra (2014), “A model of secular stagnation.” NBER Working Paper No. 20574.

Farhi, Emmanuel and Xavier Gabaix (2014), "Rare disasters and exchange rates.” NBER Working Paper No. 13805.

Foerster, Andrew T., Pierre-Daniel G. Sarte, and Mark W. Watson (2011), "Sectoral versus aggregate shocks: A structural factor analysis of industrial production." Journal of Political Economy, 119, $1-38$.

Gabaix, Xavier (2011), “The granular origins of aggregate fluctuations.” Econometrica, 79, 733-772.

Gabaix, Xavier (2012), "Variable rare disasters: An exactly solved framework for ten puzzles in macro-finance." The Quarterly Journal of Economics, 127, 645-700.

Gabaix, Xavier, Parameswaran Gopikrishnan, Vasiliki Plerou, and H. Eugene Stanley (2003), "A theory of power law distributions in financial market fluctuations.” Nature, 423, 267-230.

Gabaix, Xavier, Parameswaran Gopikrishnan, Vasiliki Plerou, and H. Eugene Stanley (2006), "Institutional investors and stock market volatility." The Quarterly Journal of Economics, 121, 461-504.

Gourio, François (2012), “Disaster risk and business cycles.” American Economic Review, 102, 27342766.

Grimmett, Geoffrey and David Stirzaker (2001), Probability and Random Processes, third edition. Oxford University Press, New York, NY.

Horvath, Michael (1998), "Cyclicality and sectoral linkages: Aggregate fluctuations from sectoral shocks.” Review of Economic Dynamics, 1, 781-808.

Horvath, Michael (2000), "Sectoral shocks and aggregate fluctuations." Journal of Monetary Economics, 45, 69-106.

Hulten, Charles R. (1978), "Growth accounting with intermediate inputs." The Review of Economic Studies, 45, 511-518.

Ibragimov, Rustam, Dwight Jaffee, and Johan Walden (2009), "Nondiversification traps in catastrophe insurance markets.” Review of Financial Studies, 22, 959-993. 
Jovanovic, Boyan (1987), "Micro shocks and aggregate risk.” The Quarterly Journal of Economics, 102, 395-409.

Kehoe, Timothy J. and Edward C. Prescott (2002), "Great Depressions of the 20th century." Review of Economic Dynamics, 5, 1-18.

Long, John B. and Charles I. Plosser (1983), “Real business cycles.” Journal of Political Economy, 91, 39-69.

Lucas, Robert E. (1977), “Understanding business cycles.” Carnegie-Rochester Conference Series on Public Policy, 5, 7 - 29.

Nakamura, Emi, Jón Steinsson, Robert Barro, and José Ursúa (2013), "Crises and recoveries in an empirical model of consumption disasters.” American Economic Journal: Macroeconomics, 5, 3574.

Rietz, Thomas A. (1988), “The equity risk premium: A solution.” Journal of Monetary Economics, 22, $117-131$.

Teicher, Henry (1984), "Exponential bounds for large deviations of sums of unbounded random variables." Sankhyā: The Indian Journal of Statistics, Series A, 46, 41-53.

Zolotarev, Vladimir M (1986), One-Dimensional Stable Distributions. American Mathematical Society, Providence, RI. 